\title{
General practice patients starting treatment for substance use problems: observations from two data sources across levels of care
}

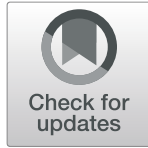

Nicole Boffin ${ }^{*}$ D, Jerome Antoine, Luk Van Baelen, Sarah Moreels and Kris Doggen

\begin{abstract}
Background: In Belgium, the incidence of treatment episodes for substance use problems is monitored by the Network of Sentinel General Practices (SGP), and at higher, specialist care levels by the Treatment Demand Indicator (TDI) surveillance. Using both data sources, we examine 1) how patients starting specialist treatment for substance use problems on referral by their GP compare to those that were referred by non-GP caregivers; 2) how patients starting GP treatment for substance use problems without receiving concurrent specialist treatment compare to those who did.

Methods: Both surveillances are based on the TDI protocol for reporting data to the European Monitoring Centre for Drugs and Drug Addiction (EMCDDA) on individuals starting treatment as a result of their substance use. Data from 2016 and 2017 were examined using 95\% confidence intervals and multivariate logistic regression.

Results: According to TDI-data $(n=16,543)$, determinants of being referred by a GP (versus by a non-GP caregiver) for specialist treatment were age $\geq$ median (OR 1.25; 95\% Cl 1.13-1.38), education $\geq$ secondary level (OR 1.27; 95\% Cl 1.15-1.41), recent employment (OR 1.71; 1.56-1.88), recent stable accommodation (3.62; 95\% Cl 3.08-4.26), first treatment episode (OR 1.72; 95\% Cl 1.57-1.87), recent daily primary substance use (OR 1.46; 95\% Cl 1.33-1.59) and mono substance use (OR 1.23; 95\% Cl 1.04-1.48). Type of substance use was a significant determinant with higher odds of using pharmaceuticals (and alcohol) (OR 1.24; 95\% Cl 1.04-1.48), and lower odds of using cannabis only/ primarily (OR 0.73; 95\% Cl 0.62-0.86), with reference to street drugs minus cannabis only/primarily. According to SGP data $(n=314)$, determinants of starting GP treatment without concurrent specialist treatment were recent employment (OR 2.58; 95\% Cl 1.36-4.91), first treatment episode (OR 2.78; 95\% Cl 1.39-5.55) and living in the Brussels or Walloon region (OR 1.97; 95\% Cl 1.06-3.66).
\end{abstract}

Conclusions: This study provides a useful insight into the general practice population treated for substance use problems. It shows that both surveillances consistently found a relatively favourable profile of general practice patients with substance use problems.

Keywords: Substance-related disorders, Public health surveillance, Patient care

\footnotetext{
* Correspondence: nicole.boffin@sciensano.be

Department of Epidemiology and Public Health, Sciensano, Brussels, Belgium
}

(c) The Author(s). 2020 Open Access This article is licensed under a Creative Commons Attribution 4.0 International License, which permits use, sharing, adaptation, distribution and reproduction in any medium or format, as long as you give appropriate credit to the original author(s) and the source, provide a link to the Creative Commons licence, and indicate if changes were made. The images or other third party material in this article are included in the article's Creative Commons licence, unless indicated otherwise in a credit line to the material. If material is not included in the article's Creative Commons licence and your intended use is not permitted by statutory regulation or exceeds the permitted use, you will need to obtain permission directly from the copyright holder. To view a copy of this licence, visit http://creativecommons.org/licenses/by/4.0/ The Creative Commons Public Domain Dedication waiver (http://creativecommons.org/publicdomain/zero/1.0/) applies to the data made available in this article, unless otherwise stated in a credit line to the data. 


\section{Background}

In many European countries the epidemiology of substance use problems is described using data about the population that started treatment for those problems. The main reason is that traditional population surveys are less reliable as far as the use of street drugs and alcohol is concerned. The Treatment Demand Indicator (TDI) was implemented on behalf of the European Monitoring Centre for Drugs and Drug Addiction (EMCDDA) to collect data in a standardized way in the European Union, Turkey and Norway [1].

Belgium started collecting TDI data from 2011 on, including also data from patients with alcohol problems only or primarily [2]. The Belgian TDI covers specialist treatment for substance use problems, excluding GP treatment because of feasibility issues. It is wellestablished that GPs do provide care to patients with substance use problems. In 2001-2 the European Study of the Epidemiology of Mental Disorders (ESEMeD) found that $17 \%$ of the Belgian population with an alcohol disorder had been receiving professional care [3]. GPs were consulted most often: $66 \%$ of people seeking care for an alcohol disorder consulted a GP and 10\% consulted only a GP [3]. GPs play a key role in Belgian healthcare, even though patients are basically free to consult a care provider of their choice. Overall, 95\% of the general population in Belgium has a regular GP [4].In 2016, following a successful pilot study, the surveillance of new treatment episodes for substance use problems was taken up by the Network of Sentinel General Practices (SGP), using an adapted TDI protocol [5]. Having acquired data from both surveillance systems over a period of 2 years (2016-2017), we decided to examine to what extent general practice patients data from both sources are in agreement and whether differences are plausible or consistent with the body of evidence, e.g. health problems presented in primary care are less severe/complex than those at higher, specialist care levels [6]. Comparing data sources is one way to explore the quality of data and may yield additional information for health policy. Our assumption is that the population starting GP treatment for substance use disorders shares core characteristics with the population referred by a GP for specialist treatment. In line with that reasoning, we assumed that general practice patients receiving mixed treatment share more traits with patients starting specialist treatment than they do with patients starting GP treatment without receiving concurrent specialist treatment. To minimize population heterogeneity, we limited the TDI study population to individuals who were referred by caregivers to start specialist treatment, in other words, individuals who had already sought professional care for their substance use problems.Using unpooled data from the SGP and TDI surveillances, this study examines 1) how patients starting specialist treatment for substance use problems on referral by their GP compare to similar patients referred by non-GP caregivers; and 2) how patients starting GP treatment for substance use problems without receiving concurrent specialist treatment compare to those who did.

\section{Methods}

Key methodological features of the TDI and SGP surveillance studies are summarised in Table 1.

\section{Settings and study participants}

The TDI and the SGP surveillance systems of providerreported, care-based data are managed within Sciensano, the Belgian Institute for Health.

The Belgian Network of SGP consists of a sample of GPs who report the occurrence and characteristics of well-defined health-related events in their daily practice [7]. Data are reported weekly on standard forms for a period of at least 1 year. Across the study period, the network comprised 125 practices. Annual statistics showed that sentinel GPs are comparable to non-sentinel GPs for age and gender. The network covers about $1,5 \%$ of the Belgian population in most Belgian districts [8]. As Belgian GPs do not serve a defined practice population, the size of the SGP patient population - the denominator - is estimated by applying the ratio of patient contacts across the entire Belgian population to the sum of weekly patient contacts in the network.The Belgian TDI study protocol has recently been described in detail [2]. The TDI register collects socio-demographic, treatment- and substance-related data about patients who started treatment for substance use disorders in a wide range of settings. Patients are interviewed by health professionals

Table 1 Key methodological features of the TDI and SGP surveillance studies

\begin{tabular}{|c|c|c|}
\hline & SGP & TDI \\
\hline Setting & General practice (primary care) & Specialist care for substance use problems (secondary and tertiairy care) \\
\hline Study design & $\begin{array}{l}\text { Sentinel surveillance by a sample of } \\
\text { general practices }\end{array}$ & Register completed by caregivers from participating centres \\
\hline $\begin{array}{l}\text { Data collection } \\
\text { tools }\end{array}$ & \multicolumn{2}{|c|}{ Standard patient data form/questionnaire to complete by caregivers for every patient starting a new treatment episode } \\
\hline Data transfer & Postal mail, data entry by Sciensano & $\begin{array}{l}\text { Online standard forms to transfer data record by record, or, repository tool to transfer } \\
\text { patient data files for a given year }\end{array}$ \\
\hline
\end{tabular}


by means of a structured questionnaire. In order to detect multiple treatment episodes in the same patients, national identification numbers are used in accordance with the European General Data Protection Regulation. Data are gathered by Sciensano using a reporting module or a repository tool allowing batch transfer of data.

Participation in the TDI surveillance is only mandatory for particular types of treatment centres for substance use problems. Throughout the study period, 221 treatment centres reported cases. TDI data from 2014 show that the best participation rates were reached by centres that are specialised in substance use problems (56 to 100\% of eligible centres) [2]. Participation rates were lower among hospitals and centres that also offer treatment for other mental health problems (17 to $52 \%$ of eligible centres).

In contrast to the SGP surveillance, the TDI surveillance is unable to estimate the incidence of treatment episodes for substance use problems in the total population for lack of data about the size of the denominator, that is the population covered by the register.

\section{Patient population samples}

To address our first research question, we selected data about the TDI patients that were referred or encouraged to seek specialist treatment by caregivers. To address our second research question, we included data from all the SGP patients.

\section{Case definitions}

The SGP instructions were based on the Belgian TDI protocol [2]. A new treatment episode starts with the first face-to-face contact with a GP/other caregiver for substance use problems. When the patient shows up with a similar treatment demand 6 months after the previous face-to-face contact, a new treatment episode starts. Treatment was defined as any activity directly targeting patients with substance use problems in order to ameliorate their mental, medical or social status. We explicitly described possible GP interventions aimed at reducing substance-related harm in active users, detoxification or abstinence, medical and non-medical problems, informal advice, counselling and support (e.g. a brief intervention). Excluded were interventions only targeting the physical consequences of substance use (e.g. infections or overdoses) or focusing mainly on problems other than substance use.

\section{Variables}

The variables in this paper (all described in Table 2) are (derived from) the items 1-6, 9-12, 14-15 and 17 of the TDI protocol 3.0 [1]. In the context of employment, accommodation and use of primary/only substance, 'recent' was understood as the last 30 days before the start of the new treatment episode. Patients who had recently been living at different places (friends' home, street, shelters, etc.) or moved from one place to another, were considered as residing in unstable accommodation. Four variables were not recorded by the SGP, respectively treatment centre type, source of referral, highest educational level completed and recent accommodation. The variable 'primary drug' was reported in less detail by the SGP, e.g. the groups 'cocaine or crack' and 'cannabis' comprise three subcategories in the TDI. One additional variable was reported by the SGP, i.e. whether or not the patient was concurrently receiving specialist treatment for substance use problems.

We summarized the type of substance use into mutually exclusive groups to fit the observed use across settings. Group I covers the use of alcohol only. Group II spans the use of pharmaceuticals, i.e. hypnotics, sedatives or pharmaceutical opioids, i.e. mainly opioid analgesics. Group III encompasses the use of street drugs, i.e. opiates, cocaine, stimulants other than cocaine, cannabis, hallucinogens and volatile inhalants. Group III was divided into two groups with group III-a spanning the use of cannabis only or primarily. Group III-b contains any other use of street drugs but no primary cannabis use and is further described as 'street drugs minus cannabis primarily'. The classification of the three groups is hierarchical in the sense that the use of pharmaceuticals (group II) may be combined with alcohol (group I), while the use of street drugs (group III) may be combined with alcohol (group I) and pharmaceuticals (group II). Methadone, buprenorphine and fentanyl were classified in group III.

\section{Analysis}

All data are episode-based. We used 95\% proportion confidence intervals $(\mathrm{CI})$ to describe patient population characteristics and bivariate associations. We used stepwise backward multiple logistic regression analysis to examine the research questions. Patient population characteristics that were significantly $(p<0.05)$ associated with the dependent variables were included in the full models. We accounted for clustering of patients within practices or treatment centres by using robust standard errors. Interaction effects between independent variables were tested only in the multivariable logistic model examining the second research question. Data were analysed with Stata 15.

\section{Results}

\section{Sample description}

The TDI register covered 60,310 episodes and the SGP network reported 314 episodes. A sample of 48,312 TDI episodes with a national identification number (NIN) showed that $28.7 \%$ of patients had more than one treatment period. No NIN are used by the SGP but proxy- 
Table 2 Characteristics of new treatment episodes of substance use problems by data source: the TDI subpopulation referred/ motivated by caregivers and the SGP population, Belgium 2016-7

\begin{tabular}{|c|c|c|c|c|}
\hline & \multicolumn{2}{|c|}{ TDI subpopulation of patients referred by caregivers $(N=16,543)$} & \multicolumn{2}{|c|}{ SGP population $(N=314)$} \\
\hline & $\mathrm{n} /$ valid $\mathrm{N}$ & $\%$ & $\mathrm{n} /$ valid $\mathrm{N}$ & $\%$ \\
\hline \multicolumn{5}{|l|}{ Sex } \\
\hline Man & $11,476 / 16,543$ & 69.5 & $220 / 314$ & 70.1 \\
\hline \multicolumn{5}{|l|}{ Age } \\
\hline$<20$ & $674 / 16,543$ & 4.1 & $14 / 312$ & 4.5 \\
\hline $20-29$ & $2982 / 16,543$ & 18.1 & $35 / 312$ & 11.2 \\
\hline $30-39$ & $4518 / 16,543$ & 27.4 & $67 / 312$ & 21.5 \\
\hline $40+$ & $8327 / 16,543$ & 50.5 & $196 / 312$ & 62.8 \\
\hline \multicolumn{5}{|l|}{ Highest educational level completed } \\
\hline None or primary & $3865 / 13,877$ & 27.9 & & \\
\hline Secondary & $7519 / 13,877$ & 54.2 & & \\
\hline Tertiary & $2493 / 13,877$ & 18.0 & & \\
\hline Recent stable accommodation & $13,075 / 16,219$ & 80.6 & & \\
\hline Recently employed & $3394 / 14,989$ & 22.6 & $135 / 292$ & 46.2 \\
\hline \multicolumn{5}{|l|}{ Region } \\
\hline Flanders & $9936 / 16,543$ & 60.0 & $198 / 314$ & 63.1 \\
\hline Wallonia & $4495 / 16,543$ & 27.1 & $82 / 314$ & 26.1 \\
\hline Brussels & $2112 / 16,543$ & 12.8 & $34 / 314$ & 10.8 \\
\hline Previous treatment & $10,952 / 16,039$ & 68.3 & $178 / 282$ & 63.1 \\
\hline \multicolumn{5}{|l|}{ Type of substance use } \\
\hline Alcohol only (I) & $7354 / 16,543$ & 44.5 & $176 / 314$ & 56.1 \\
\hline Pharmaceuticals (and alcohol) (II) & $1047 / 16,543$ & 6.3 & $46 / 314$ & 14.7 \\
\hline Cannabis only/primarily (III-a) & $2077 / 16,543$ & 12.6 & $30 / 314$ & 9.6 \\
\hline Street drugs minus cannabis primarily (III-b) & $6065 / 16,543$ & 36.7 & $62 / 314$ & 19.8 \\
\hline Mono-substance use & $10,427 / 16,543$ & 63.0 & $254 / 314$ & 80.9 \\
\hline \multicolumn{5}{|l|}{ Recent use of primary/only substance } \\
\hline No use in last 30 days & $2193 / 15,576$ & 14.1 & $12 / 258$ & 4.7 \\
\hline$\leq 1$ day a week & $923 / 15,576$ & 5.9 & $8 / 258$ & 3.1 \\
\hline 2-3 days a week & $1386 / 15,576$ & 8.9 & $16 / 258$ & 6.2 \\
\hline 4-6 days/week & $1775 / 15,576$ & 11.4 & $22 / 258$ & 8.5 \\
\hline Daily & $9299 / 15,576$ & 59.7 & $200 / 258$ & 77.5 \\
\hline \multicolumn{5}{|l|}{ Type of treatment } \\
\hline Outpatient treatment & $5254 / 16,543$ & 31.8 & & \\
\hline \multicolumn{5}{|l|}{ Inpatient treatment: } \\
\hline Inpatient, non-hospital & $2064 / 16,543$ & 12.5 & & \\
\hline Psychiatric hospital & $3897 / 16,543$ & 23.6 & & \\
\hline General hospital (psychiatric service) & $5220 / 16,543$ & 31.6 & & \\
\hline Treatment for criminal law offenders & $108 / 16,543$ & 0.7 & & \\
\hline \multicolumn{5}{|l|}{ Source of referral } \\
\hline GP & $4515 / 16,543$ & 27.3 & & \\
\hline Care services for substance use problems & $2349 / 16,543$ & 14.2 & & \\
\hline Hospital & $5277 / 16,543$ & 31.9 & & \\
\hline Medical-psycho-social services & $4402 / 16,543$ & 26.6 & & \\
\hline
\end{tabular}


indicators (age, sex, ...) revealed 11 patients $(3.6 \%)$ with more than one treatment period.For the first research question, we excluded TDI episodes from self-referred patients $(n=26,950 ; 46.0 \%)$, motivated by peers $(n=8881$; $15.2 \%)$ or by court, probation or police $(n=6152 ; 10.5 \%)$. Excluding 33 episodes with invalid substance use data, we thus included 16,543 of 60,310 (27.4\%) TDI episodes concerning patients that were referred by caregivers. Four types of referring caregivers were distinguished: GPs $(n=4515,27.3 \%)$, care services for substance use problems $(n=2349,14.2 \%)$, general or psychiatric hospitals $(n=5277,31.9 \%)$ and (other) medicalpsycho-social services $(n=4402,26 ; 6 \%)$.

Table 2 describes the characteristics of 16,543 treatment episodes reported to the TDI and the characteristics of 314 treatment episodes reported by the SGP network. The median age of the first use of the primary or only problem substance among TDI patients was 17 (Interquartile range (IQR): 15-21) and 18 (IQR 16-25) among SGP patients. In both populations most patients had alcohol problems only. Compared to the SGP population, the use of street drugs was higher in the TDI population, especially street drugs minus cannabis primarily (group III-b), while the use of pharmaceuticals (and alcohol) (group II) was lower. More than half (53.5\%) of hypnotics and sedatives in the TDI population was used together with street drugs and thus classified in group III. In the SGP population pharmaceuticals were mostly combined with alcohol $(70.8 \%)$ and thus classified in group II.

How do patients starting specialist treatment for substance use problems on referral by their GP compare to similar patients referred by non-GP caregivers?

All socio-demographic and substance use characteristics of GP-referred patients and patients referred by other caregivers were significantly different (Table 3 ). GP-referral was also associated with type of treatment: almost half of the GP-referred patients started general hospital-based treatment, while GP-referral to inpatient treatment outside the hospital setting was rare. In the Walloon region, relatively more patients were referred by their GP and relatively fewer in the Brussels region.

Socio-demographic determinants of being referred by a GP were: higher age, higher education, recent employment and stable accommodation. Substance use determinants of a GP-referral were: first treatment episode, recent primary substance use and mono-substance use. Using street drugs minus cannabis primarily (III-b) as a reference category, GP-referred patients had (borderline) higher odds for using alcohol only (I) or pharmaceuticals (and alcohol) (II) and lower odds for using cannabis only/ primarily (III-a).
How do patients starting GP treatment for substance use problems without receiving concurrent specialist treatment compare to those who did?

For 9 out of 314 patients it was unknown whether they were receiving specialist treatment at the start of a new GP treatment episode, while $27.9 \%$ (85 of 305) patients did so. For 13 out of 85 patients the type of treatment was not reported, 53 patients received outpatient treatment and 19 inpatient treatment.

Table 4 shows significantly different socio-demographic and substance use characteristics between patients starting GP treatment only and patients who were concurrently receiving specialist treatment. Determinants of receiving GP treatment without concurrent specialist treatment were no previous treatment episode, being at work and living in the Walloon or Brussels region.

\section{Discussion}

Our main findings are twofold. First, the TDI data show that patients starting specialist treatment for substance use problems on referral by their GP have a distinct, more favourable profile compared to patients who were referred by non-GP caregivers. They were relatively older and socially better-off considering their education, employment status and their stable housing status. They were also better off regarding substance use problems with relatively more problems of alcohol and/or pharmaceuticals, more mono-substance use and first treatment episodes. There is some evidence that problem use of alcohol alone is less severe than poly substance use or street drug use $[9,10]$. Second, the SGP showed that patients starting GP treatment without receiving specialist treatment were also better off compared to similar patients receiving concurrent specialist treatment. Among the latter, fewer patients were recently employed and more had been in treatment before. We thus found evidence confirming our assumption that patients starting GP treatment and concurrently receive specialist treatment are more similar to GPreferred patients starting specialist treatment compared to patients starting GP treatment only.

This study found considerable agreement between two data sources about general practice patients with substance use problems. New knowledge was acquired about the (referred) general practice population, such as education and recent accommodation. Yet, nearly 13\% of educational data were missing in the TDI.

Our study has other weaknesses. One limitation is that data were compared on an aggregated level. So far, it has been impossible to measure overlap between the two surveillance systems at the patient level by unique patient identifiers. Due to the cross-sectional design of both the studies this paper presents a mere snapshot of the populations at a given point in time. Consequently, we cannot tell whether patients started GP treatment 
Table 3 New episodes of specialist treatment for substance use problems by source of referral, TDI surveillance, Belgium 2016-7 $(N=16,543)$

\begin{tabular}{|c|c|c|c|c|c|}
\hline & \multicolumn{2}{|c|}{$\begin{array}{l}\text { Non-GP caregiver } \\
n=12,028\end{array}$} & \multicolumn{2}{|l|}{$\begin{array}{l}\mathrm{GP} \\
n=4515\end{array}$} & \multirow{2}{*}{$\begin{array}{l}\text { Adjusted OR }(95 \% \mathrm{Cl}) \text { for } \\
\text { referral by GP (versus non-GP } \\
\text { caregiver) }(n=12,032)\end{array}$} \\
\hline & $\mathrm{n} / \mathrm{N}$ & $\%(95 \% \mathrm{Cl})$ & $\mathrm{n} / \mathrm{N}$ & $\%(95 \% \mathrm{Cl})$ & \\
\hline Sex: man & $8489 / 12,013$ & $70.7(69.8-71.5)$ & $2987 / 4510$ & $66.2(64.8-67.6)$ & Removed $^{\mathrm{b}}$ \\
\hline Age $\geq$ median & $5659 / 11,999$ & $47.2(46.3-48.1)$ & $2668 / 4507$ & $59.2(57.7-60.6)$ & $1.25(1.13-1.38)$ \\
\hline Secondary educational level or higher & $6840 / 9896$ & $69,1(68.2-70.0)$ & $3172 / 3981$ & 79,7 (78.4-80.9) & $1.27(1.15-1.41)$ \\
\hline Recently employed & $2000 / 10,788$ & $18.5(17.8-19.3)$ & $1394 / 4201$ & $33.2(31.8-34.6)$ & $1.71(1.56-1.88)$ \\
\hline Recent stable accommodation & $8846 / 11,736$ & $75.4(74.6-76.2)$ & $4229 / 4483$ & $94.3(93.6-95.0)$ & $3.62(3.08-4.26)$ \\
\hline First treatment & $3183 / 11,654$ & $27.3(26.5-28.1)$ & $1904 / 4385$ & $43.4(41.9-44.9)$ & $1.72(1.57-1.87)$ \\
\hline \multicolumn{6}{|l|}{ Type of substance use } \\
\hline $\begin{array}{l}\text { Street drugs minus cannabis } \\
\text { primarily (III-b) }\end{array}$ & $4748 / 12,028$ & $39.5(38.5-40.3)$ & $1174 / 4515$ & $26.0(24.7-27.3)$ & reference \\
\hline Alcohol only (I) & $4827 / 12,028$ & $40.1(39.3-41.0)$ & $2527 / 4515$ & $56.0(54.5-57.4)$ & $1.10(0.94-1.29)$ \\
\hline Pharmaceuticals (and alcohol) (II) & $799 / 12,028$ & $6.6(6.2-7.1)$ & $391 / 4515$ & $8.7(7.9-9.5)$ & $1.24(1.04-1.48)$ \\
\hline Cannabis only/primarily (III-a) & $1654 / 12,028$ & $13.8(13.1-14.4)$ & $423 / 4515$ & $9.4(8.5-10.3)$ & $0.73(0.62-0.86)$ \\
\hline Mono substance use & $7155 / 12,028$ & $59.5(58.6-60.4)$ & $3272 / 4515$ & $72.5(71.1-73.8)$ & $1.23(1.04-1.48)$ \\
\hline $\begin{array}{l}\text { First use of primary/only substance } \\
\text { at } \geq 17 \text { years }\end{array}$ & $4668 / 8475$ & $55.1(54.0-56.1)$ & $1896 / 3067$ & $61.8(60.1-63.5)$ & a \\
\hline $\begin{array}{l}\text { Recent daily use of primary/only } \\
\text { substance }\end{array}$ & $6372 / 11,308$ & $56.3(55.4-57.3)$ & $2927 / 4268$ & $68.6(67.2-70.0)$ & $1.46(1.33-1.59)$ \\
\hline Type of treatment & & & & & a \\
\hline Outpatient treatment & $4022 / 11,920$ & $33.7(32.9-34.6)$ & $1232 / 4515$ & $27.3(26.0-28.6)$ & \\
\hline Inpatient, non-hospital & 1928/11,920 & $16.2(15.5-16.8)$ & $136 / 4515$ & $3.0(2.5-3.6)$ & \\
\hline Psychiatric hospital & 2914/11,920 & $24.4(23.7-25.2)$ & $983 / 4515$ & $21.8(20.6-23.0)$ & \\
\hline General hospital/psychiatry & $3056 / 11,920$ & $25.6(24.9-26.4)$ & $2164 / 4515$ & $47.9(46.5-49.4)$ & \\
\hline \multicolumn{6}{|l|}{ Region of SGP } \\
\hline Flemish & $7180 / 12,028$ & $59.7(58.8-60.6)$ & $2756 / 4515$ & $61.0(59.6-62.5)$ & Removed $^{\mathrm{b}}$ \\
\hline Walloon & $3133 / 12,028$ & $26.0(25.3-26.8)$ & $1362 / 4515$ & $30.2(28.8-31.5)$ & \\
\hline Brussels & $1715 / 12,028$ & $14.3(13.6-14.9)$ & $397 / 4515$ & $8.8(8.0-9.7)$ & \\
\hline
\end{tabular}

Non-overlapping confidence intervals are in bold

a First use of primary substance was not included in the multivariable logistic model because of the low number of valid data and its high association with the type of substance use. Type of treatment was not included in the multivariable logistic model as it is not a socio-demographic patient characteristic or a substance use characteristic

${ }^{\mathrm{b}}$ Variable was removed because it did not significantly improved the fit of the model

before and/or after starting specialist treatment. Neither do we know whether patients starting specialist treatment also receive(d) GP treatment.

Yet, the essence of a treatment episode clearly differs across levels of care. As described above (see "Sample description"), the percentage of patients with more than one treatment episode was much higher in the TDI (28.7\%) than in the SGP (3.5\%), despite uniform definitions. Several reasons may account for this large difference. In the TDI, a treatment trajectory may include subsequent treatment episodes in different settings, e.g. hospital-based detoxification first, followed by drug-free therapy in another setting. In general practice the difference between a health problem episode and a treatment episode is relatively small, especially as to unhealthy lifestyle. Moreover, the difference between new and ongoing problems and treatment is equally blurry, especially when chronic problems are concerned. In contrast to specialist treatment, substance use may not be an issue in every GP-patient contact during a treatment episode, even when the problem is still present. Conversely, the end/start of a treatment episode is much clearer in specialist treatment: when the patient fails to show up/shows up again. Given those limitations, it is impossible to estimate the size of the gap in the TDI register due to its non-coverage of general practice.

A PubMed search (September 2019) using the medical subheadings of 'substance-related disorders/epidemiology' and 'general practice' did not reveal recent papers with comparable research questions. Papers with (comparable) 
Table 4 New episodes of GP treatment for substance use problems without and with concurrent specialist treatment, SGP surveillance, Belgium 2016-7 $(N=305)^{a}$

\begin{tabular}{|c|c|c|c|c|c|}
\hline & \multicolumn{2}{|c|}{$\begin{array}{l}\text { GP treatment only (no concurrent treatment) } \\
n=220\end{array}$} & \multicolumn{2}{|c|}{$\begin{array}{l}\text { Concurrent specialist treatment } \\
n=85\end{array}$} & \multirow{2}{*}{$\begin{array}{l}\text { Adjusted OR } \\
(95 \% \mathrm{CI}) \text { for GP } \\
\text { treatment only } \\
(n=259)\end{array}$} \\
\hline & $\mathrm{n} / \mathrm{N}$ & $\%(95 \% \mathrm{Cl})$ & $\mathrm{n} / \mathrm{N}$ & $\%(95 \% \mathrm{Cl})$ & \\
\hline Recently employed & $113 / 207$ & $54.6(47.5-61.5)$ & $18 / 76$ & $23.7(14.7-34.8)$ & $2.58(1.36-4.91)$ \\
\hline First treatment & $91 / 198$ & $46.0(38.9-53.2)$ & $13 / 79$ & $16.5(9.1-26.5)$ & $2.78(1.39-5.55)$ \\
\hline \multicolumn{6}{|l|}{ Type of substance use } \\
\hline $\begin{array}{l}\text { Street drugs minus cannabis } \\
\text { primarily (III-b) }\end{array}$ & $37 / 220$ & $16.8(12.1-22.4)$ & $23 / 85$ & $27.1(18.0-37.8)$ & Removed $^{c}$ \\
\hline Mono substance use & $188 / 220$ & $85.5(80.1-89.8)$ & $60 / 85$ & $70.6(59.7-80.0)$ & Removed $^{c}$ \\
\hline Recent use of primary substance & 187/191 & $97.9(94.7-99.4)$ & $54 / 61$ & $88.5(77.8-95.3)$ & Not included ${ }^{b}$ \\
\hline \multicolumn{6}{|l|}{ Region } \\
\hline Flemish & $126 / 220$ & $57.3(50.4-63.9)$ & $64 / 85$ & $75.3(64.7-84.0)$ & Reference \\
\hline Walloon & $65 / 220$ & $29.5(23.6-36.0)$ & $16 / 85$ & $18.8(11.2-28.8)$ & $1.97(1.06-3.66)$ \\
\hline Brussels & $29 / 220$ & $13.2(9.0-18.4)$ & $5 / 85$ & $5.9(1.9-1.3)$ & \\
\hline
\end{tabular}

Sex, age and age of first use of primary substance were not significantly associated at the univariate level with receiving concurrent specialist treatment or not Non-overlapping confidence intervals are in bold

${ }^{a}$ For 9 of 314 patients it was unknown whether they were receiving concurrent specialist treatment

${ }^{b}$ Recent use of primary substance was not included in the initial multivariate logistic model due to the small number of positive cases

c Variable was removed because it did not significantly improved the fit of the model

findings from the EMCDDA TDI were not found in PubMed. The focus of the SGP pilot study was different but some core results are comparable, e.g. type of substance use, previous treatment episodes and regional differences [5]. The pilot showed that 7 months after the baseline recording of new and ongoing episodes of GPtreatment, $21 \%$ of the patients who continued GP treatment also received specialist treatment. This proportion is similar to the proportion of $28 \%$ episodes of concurrent specialist treatment we found in this study.Monitoring treatment demand in general practice is one way of dealing with the problem of underdiagnosis of substance use problems, mostly alcohol, in general practice [11]. Yet, 'detected' patients in general practice may have more severe problems resulting in a relatively higher rate of referrals and concurrent specialist treatment. Unfortunately, we did not find evidence to verify this assumption.

Our findings fit the knowledge about general practice and GP care of patients with chronic, recurrent substance use problems. According to a good health services model, GPs provide as much care as possible to patients and refer patients to specialist health facilities only when more complex care is needed [12]. Continuity is a major attribute of general practice care, comprising continuing care over a lifetime, across health conditions and levels of care. In the context of substance use problems, this means preventive care, e.g. active screening and short interventions, and, aftercare or chronic patient care, e.g. patient support in case of relapse [13]. The finding that relatively more GP-referred and GP-treated patients were using the primary substance daily in the last 30 days may be exemplary of the chronic care role of GPs towards patients with, most likely, problems of alcohol and/or pharmaceuticals. Maybe those patients seek help from their GP in times of crisis: when they are drinking too much or have relapsed into drinking. GPs may be less strict than specialist caregivers about abstinence as a condition for starting treatment, but the widespread availability of alcohol and, to a lesser degree, pharmaceuticals, possibly also play a role.

This study yielded useful information for health policy and research. We found that GPs meet the demand of a specific population with substance use problems. This population is better off in more than one way. They may prefer to seek discreet help from their GPs above having to interrupt their social/work activities and seek specialist treatment, often outside the community. In this study, a relatively small part of the TDI population was examined. We believe that further research of motivators and referring caregivers of patients starting specialist treatment for substance problems would be useful to profile the population and treatment demand.

\section{Conclusions}

We found considerable agreement between the SGP surveillance and the TDI-surveillance about the general practice population starting a new treatment episode for substance use problems in Belgium over a 2-year period. Examining the two data sources yielded new knowledge about the general practice population that is treated for substance use problems, more specifically, its relatively favourable profile. 


\section{Abbreviations}

Cl: Confidence interval; GP: General practitioner; OR: Odds ratio; SGP: Sentinel General Practices; TDI: Treatment Demand Indicator

\section{Acknowledgements}

All GPs from the Belgian Network of Sentinel General Practices are gratefully acknowledged. The Belgian Network of SGP is supported by the Flemish Agency for Care and Health and the French Federation of Brussels and the Walloon region.

\section{Authors' contributions}

NB and SM were responsible for the SGP data collection, JA was responsible for the TDI data collection. NB was responsible for the study conception, analyses and report of the study. JA, LVB and KD were involved in the critical revision of the manuscript. All authors have read and approved the final manuscript.

\section{Funding}

This study received no specific grant from any funding agency in the public, commercial or not-for-profit sectors.

\section{Availability of data and materials}

The datasets generated and/or analysed during the current study are not publicly available due to lack of resources but they are available from the corresponding author on reasonable request.

\section{Ethics approval and consent to participate}

The Belgian Network of SGP was approved as a whole by the Ethical Committees of the Scientific Society of Flemish GPs and the Catholic University of Louvain (UCL). The Belgian TDI protocol adheres to the European protocol and the Belgian privacy legislation.

\section{Consent for publication}

Not applicable.

\section{Competing interests}

The authors declare that they do not have any conflicts of interest to disclose.

Received: 23 December 2019 Accepted: 3 June 2020

Published online: 18 June 2020

\section{References}

1. Montanari L, Pirona A, Guarita B, Hedrich D, Mounteney J, Vicente J. The Experience of the Treatment Demand Indicator in Europe: A Common Monitoring Tool Across 30 Countries. J Stud Alcohol Drugs Suppl. 2019;18: 139-51. https://doi.org/10.15288/jsads.2019.s18.139.

2. Antoine J, De Ridder K, Plettinckx E, Blanckaert P, Gremeaux L. Treatment for substance use disorders: the Belgian treatment demand Indicator registration protocol. Arch Public Health. 2016;74:27.

3. Bruffaerts R, Bonnewyn A, Van Oyen H, Demarest S, Demyttenaere K. Patterns of service use for mental health disorders in Belgium. Results of the European study on epidemiology of mental disorders (ESEMeD). Rev Med Liege. 2004:59:136-44.

4. Belgian Health Interview Survey-interactive analysis HISIA. Brussels: Sciensano. https://hisia.wiv-isp.be/ (Accessed 16 Mar 2020).

5. Boffin N, Antoine J, Moreels S, Wanyama S, De Ridder K, Peremans L, et al. General practice patients treated for substance use problems: a crossnational observational study in Belgium. BMC Public Health. 2016;16:1235.

6. Green LA, Fryer GE Jr, Yawn BP, Lanier D, Dovey SM. The ecology of medical care revisited. N Engl J Med. 2001;344:2021-5.

7. Deckers JG, Paget WJ, Schellevis FG, Fleming DM. European primary care surveillance networks: their structure and operation. Fam Pract. 2006;23:151-8.

8. Boffin N. Surveillance studies of suicidal behavior and depression by the Belgian network of sentinel general practices: Ghent University; 2013.

9. Dick DM, Agrawal A, Wang JC, Hinrichs A, Bertelsen S, Bucholz KK, et al. Alcohol dependence with comorbid drug dependence: genetic and phenotypic associations suggest a more severe form of the disorder with stronger genetic contribution to risk. Addiction. 2007;102:1131-9.
10. Stinson FS, Grant BF, Dawson DA, Ruan WJ, Huang B, Saha T. Comorbidity between DSM-IV alcohol and specific drug use disorders in the United States: results from the National Epidemiologic Survey on alcohol and related conditions. Drug Alcohol Depend. 2005:80:105-16.

11. Mitchell AJ, Meader N, Bird V, Rizzo M. Clinical recognition and recording of alcohol disorders by clinicians in primary and secondary care: meta-analysis. Br J Psychiatry. 2012;201:93-100.

12. World Health Organization. Everybody business : strengthening health systems to improve health outcomes: WHO's framework for action. Geneva: World Health Organization; 2007.

13. McLellan AT, Lewis DC, O'Brien CP, Kleber HD. Drug dependence, a chronic medical illness: implications for treatment, insurance, and outcomes evaluation. JAMA. 2000;284:1689-95.

\section{Publisher's Note}

Springer Nature remains neutral with regard to jurisdictional claims in published maps and institutional affiliations.

\section{Ready to submit your research? Choose BMC and benefit from:}

- fast, convenient online submission

- thorough peer review by experienced researchers in your field

- rapid publication on acceptance

- support for research data, including large and complex data types

- gold Open Access which fosters wider collaboration and increased citations

- maximum visibility for your research: over $100 \mathrm{M}$ website views per year

At BMC, research is always in progress.

Learn more biomedcentral.com/submissions 